\title{
Toward a 3-D Camera Based on Single Photon Avalanche Diodes
}

\author{
Cristiano Niclass, Alexis Rochas, Pierre-André Besse, and Edoardo Charbon, Member, IEEE
}

\begin{abstract}
A three-dimensional (3-D) imager is presented, capable of computing the depth map as well as the intensity scale of a given scene. The heart of the system is a two-dimensional array of single photon avalanche diodes fabricated in standard CMOS technology. The diodes exhibit low-noise equivalent-power high-dynamic range, and superior linearity. The 3-D imager achieves submillimetric precision at a depth-of-field of a few meters. This precision was achieved by averaging over 10000 measurements. The imager operates using a standard laser source pulsed at $50 \mathrm{MHz}$ with $40-\mathrm{mW}$ peak power and requires no mechanical scanning mechanisms or expensive optical equipment.
\end{abstract}

Index Terms-Avalanche photodiode, CMOS photodetector, quantum architecture, single-photon counter, three-dimensional (3-D) camera.

\section{INTRODUCTION}

$\mathbf{T}$ HE EMERGENCE of novel potentially high-volume applications for three-dimensional (3-D) optical imaging has generated renewed interest in such devices. These applications include land and sea surveyors, virtual keyboards, face recognition systems, nonionising medical tomographic imagers, stage and choreography analysis tools, etc. A new generation of solidstate imagers has been designed to address concerns of compactness and speed. However, power, safety, and cost limitations still exist.

Current solid-state 3-D optical imaging is based on three main techniques: triangulation, interferometry, and time-of-flight (TOF) using modulated and pulsed laser sources. All these methods have advantages and shortcomings and have been extensively researched. In triangulation systems, distance to a precise point in the scene is derived from the angle of incidence of a known point source and the angle of reflected light, whereby a conventional optical sensor is usually capturing such reflection [1]. The main disadvantage of such systems is the speed requirement on the sensor, power dissipation, and a somewhat limited precision. Interferometry is being used for the high levels of accuracy it ensures. However, interferometers are usually bulky and very expensive [2]

Optical TOF rangefinders using highly collimated coherent light sources have been technologically feasible for decades. Such devices measure the distance and velocity of a target by calculating the time the optical ray requires to complete a round trip. This time can be measured explicitly by means of a fast

Manuscript received January 27, 2004; revised May 25, 2004. This work was supported by the Swiss National Science Foundation under Grant 620-066110.

The authors are with the Swiss Federal Institute of Technology, Lausanne 1015, Switzerland.

Digital Object Identifier 10.1109/JSTQE.2004.833886 chronometer. An implicit measurement is also possible by evaluating the phase difference between a modulated light source and the reflected light from the target [3].

To achieve submillimetric accuracies: 1) subpicosecond time discrimination capability, 2) high-detection sensitivity, and 3) low-ray divergence are necessary.

In modulation-type imagers, time accuracy can be relaxed by use of high-modulation frequencies in conjunction with homodyne phase discrimination at the pixel level [3], [4]. However, relatively powerful laser or LED sources are still required and accuracy is limited by the speed at which the sensor can be clocked. Moreover, with the increase of clock frequency potentially complex methods must be devised to resolve the ambiguity of phase measurements and to compensate for depth-of-field reduction.

Several 3-D imagers based on the pulsed method can be found in the literature [5]-[7]. In [5], a long-distance rangefinder is presented based on an array of single photon avalanche diodes (SPADs) fabricated in an ad hoc silicon technology. To enable high-performance time discrimination on a pixel basis, a second chip was fabricated in standard CMOS consisting of an array of counters aligned with the SPADs. A technique, known as bridge bonding, was used to allow the SPADs to be aligned and contacted each with the corresponding circuitry. The main disadvantage of this approach is the need for hybrid technology and nonstandard bonding techniques.

A single detector with mechanical scanning device was proposed in [6]. In this approach, the specification on the optomechanical setup is generally very stringent and often leads to relatively compact but complex devices.

In [7], a conventional CMOS array was designed in which shutter speed and electrical amplification had been maximized. High peak light source power was still required, even at relatively short ranges, thus making the approach impractical for potential human hazard considerations. Moreover, the power dissipated by the device, excluding the power source, was significant, thus preempting its use in most mobile applications.

In this paper, a new solid-state 3-D imager is presented based on the pulsed method, which requires a potentially inexpensive laser source with milliwatt peak power. The sensor was designed for short-range applications, such as face recognition, virtual user interfaces, medical tomography, etc. These applications generally require a range of less than $10 \mathrm{~m}$.

The sensor consists of an array of SPADs, implemented in standard CMOS technology, sequentially performing 32 distance measurements with submillimetric precision. Note that the precision or accuracy of a time or a distance is defined as the uncertainty of the measurement. A time precision of a few tens of 


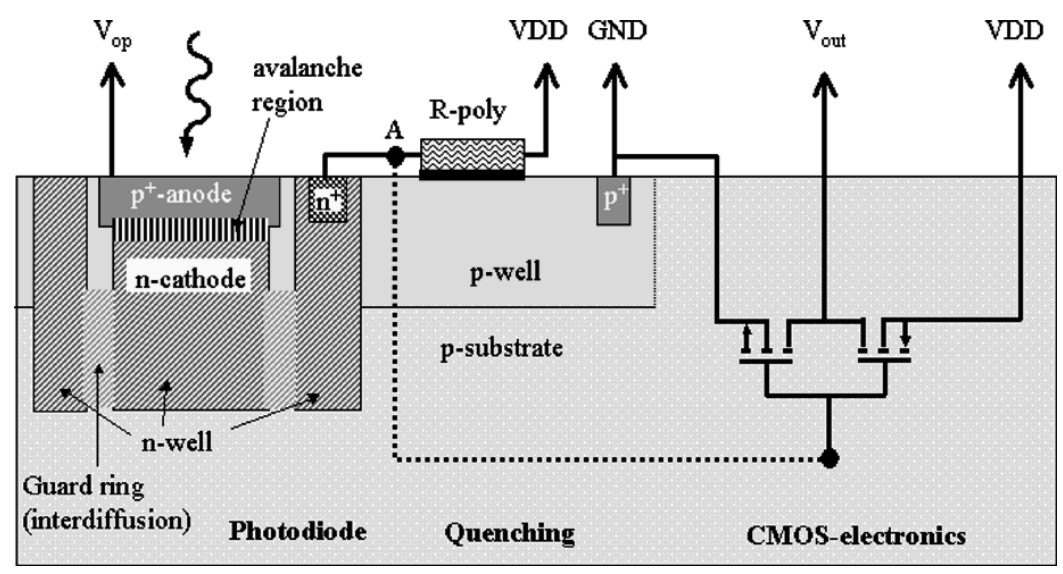

Fig. 1. Pixel schematic.

picoseconds was made possible by the jitter properties of SPADs when operating in Geiger mode. Mechanical scanning could be eliminated by illumination of the scene with a cone of light that is reflected by the surface and reaches every pixel almost simultaneously. The cone of light could be obtained by simply uncollimating the laser source.

The 3-D camera system is capable of characterizing the optical field by mapping both the depth of the scene as well as the intensity of the light reflected by it. Extremely tight speed and accuracy specifications are met, while keeping power dissipation to a minimum, thanks to the inherent simplicity of the sensor design. Moreover, due to the reduced power of the laser source, it is possible to guarantee strict eye safety operation of the system even with a duty cycle of $100 \%$.

Alternatively, with a significant increase of optical peak power it is possible to use time gating techniques to discriminate ballistic and near-ballistic photons from scattering ones, thus providing means to perform transillumination type bioanalysis. An imager based on a SPAD matrix is a good candidate for this application as it combines picosecond time precision and high sensitivity to weak rays as they traverse human tissue. Due to the nonionising nature of light, the biological impact of this type of imaging technique is, conceivably, orders of magnitude smaller than that of X-rays, nuclear particle streams, etc. [8].

To date, the 3-D imager system presented in this paper is not yet completely integrated. Some of the components used in the time discrimination procedure are discrete. Therefore, some of the performance measurements could be improved with further component integration.

The paper is organized as follows. The principle of operation of the SPADs is explained in detail in Section II. The 3-D camera system and measurements are outlined in Section III. In Section IV, we evaluate the sensor based on SPADs for twodimensional (2-D) grayscale images.

\section{Pixel Design, Operation, And Characteristics}

The 3-D camera proposed in this paper is composed of an $8 \times 4$ array of pixels. Each pixel is comprised of a SPAD and quenching electronics. This section outlines the pixel design principles and measured characteristics. More details can be found in [9].

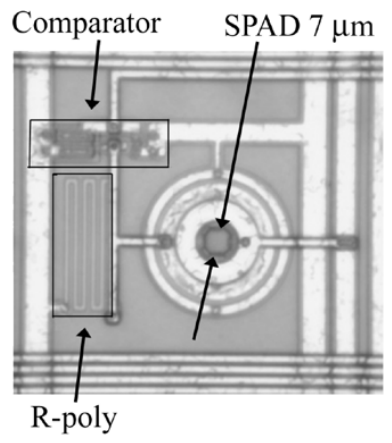

Fig. 2. Photomicrograph of a pixel.

Fig. 1 shows the cross section and schematic of the diode and read-out circuitry. Quenching resistor and comparator are cointegrated in the pixel. As a result, parasitic capacitances can be reduced and large pixel arrays can be built. The avalanche multiplication takes place in the active $\mathrm{p}+/ \mathrm{n}$-well junction. Premature edge breakdown is prevented by a guard ring, which is obtained by interdiffusion of two n-wells. This structure requires no extra masks or postprocessing steps and can be fabricated in a standard CMOS process [10].

The pixel, shown in the photomicrograph of Fig. 2, consists of a circular SPAD of $7 \mu \mathrm{m}$ in diameter, a quenching resistor R-poly in series between the cathode of the diode and VDD, and a comparator that transforms the Geiger voltage pulse at node A onto a digital signal. $\mathrm{V}_{\mathrm{OP}}$ is used to bias the anode at $-18.5 \mathrm{~V}$ while VDD $=5 \mathrm{~V}$ powers the remainder of the circuit. The breakdown voltage $\mathrm{V}_{\mathrm{bd}}$ of the SPAD is $21 \mathrm{~V}$, thus the diode is biased with an excess voltage above breakdown $\mathrm{V}_{\mathrm{e}}=\left|\mathrm{V}_{\mathrm{OP}}\right|+$ $\mathrm{VDD}-\mathrm{V}_{\mathrm{bd}}=2.5 \mathrm{~V}$. The comparator is implemented as a simple inverter with a threshold voltage of $3 \mathrm{~V}$.

The current pulse generation can be explained in the following way [9]. When a photon reaches the avalanche region, a primary electron-hole pair is generated. The avalanche process begins and a strong current flows in the junction, causing a voltage drop across R-poly. The voltage $\mathrm{V}_{\mathrm{A}}$ at node $\mathrm{A}$ (Fig. 1) drops from VDD to VDD $-V_{e}$, switching the inverter logic state and stopping the avalanche. Once the avalanche current is quenched, the parasitic capacitance starts to recharge, and the voltage $\mathrm{V}_{\mathrm{A}}$ increases again to VDD. The total time for the 


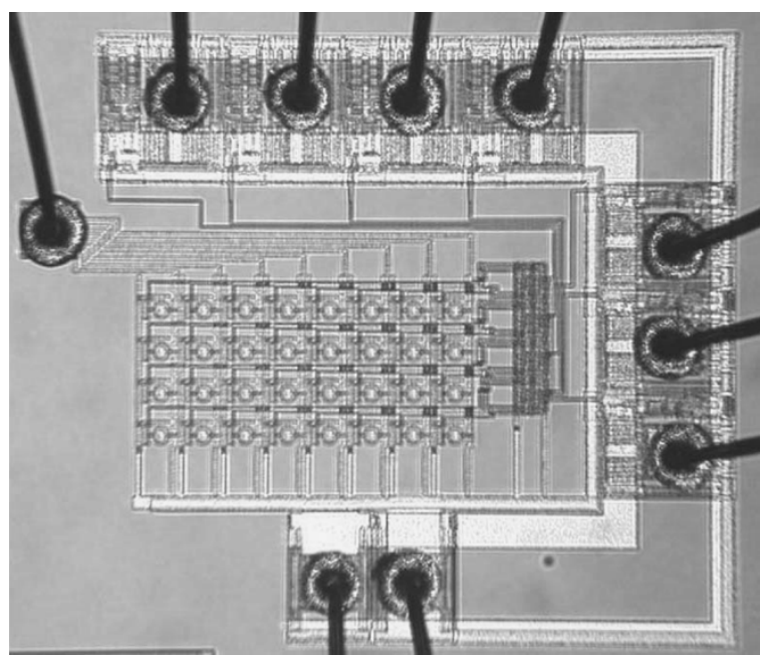

Fig. 3. Bonded $8 \times 4$ SPAD array.

quenching and recharging processes determines the lowest possible time interval between detectable photons. This time constant is known as dead time.

The array of SPADs (Fig. 3) was fabricated using the standard $0.8 \mu \mathrm{m}$ CMOS technology. For each pixel the parasitic capacitance is about $70 \mathrm{fF}$, the quenching resistor is $270 \mathrm{k} \Omega$, leading to a dead time of $32 \mathrm{~ns}$ [11]. Furthermore, the SPAD has a quantum efficiency $\eta$ of about $10 \%$ at $635 \mathrm{~nm}$, is free of after pulses, and exhibits a Dark Count Rate (DCR) of about $50 \mathrm{~Hz}$ and a time jitter of $50 \mathrm{ps}$. At a pitch of $75 \mu \mathrm{m}$ no inter-SPAD cross talk was detectable. Despite the small number of pixels, the above array is a demonstrator of a larger 3-D camera.

\section{RANGEFINDER}

A rangefinder based on an $8 \times 4$ SPAD array was designed and implemented [12]. Fig. 4 shows the architecture of the complete 3-D camera, which includes classic row and column selection circuits identical to conventional CMOS imagers. Unlike conventional CMOS imagers, however, the column readout circuitry requires no amplification, no analog processing, no sample-and-hold, and no A/D conversion before being routed outside the chip. This is due to the fact that the output of a SPAD is essentially a digital signal.

Moreover, as a direct consequence of design simplicity, the power dissipation of the sensor is very low. In our experiments, it fell below the resolution of instrumentation, dominated by other external sources that cumulatively amounted to $1 \mathrm{~mW}$.

A digital multiplexer and row/column decoders were programmed to facilitate random access of a pixel output. External bias voltage sources were used, even though such voltages can and will be generated on-chip in a next-generation design. Fig. 5 shows the electrooptical setup of the 3-D camera.

The distance between the laser source and the points in the scene is computed by measuring the TOF $\tau$ of a light beam from source to target. The actual distance $d$ is computed as $d=c \tau / 2$, where $c$ is the speed of light. A 635-nm laser diode, pulsed at repetition rate $f_{R}=50 \mathrm{MHz}$, with pulse width $T_{P}=100 \mathrm{ps}$ and peak power $\hat{P}_{S}=40 \mathrm{~mW}$ is directed toward the target. The

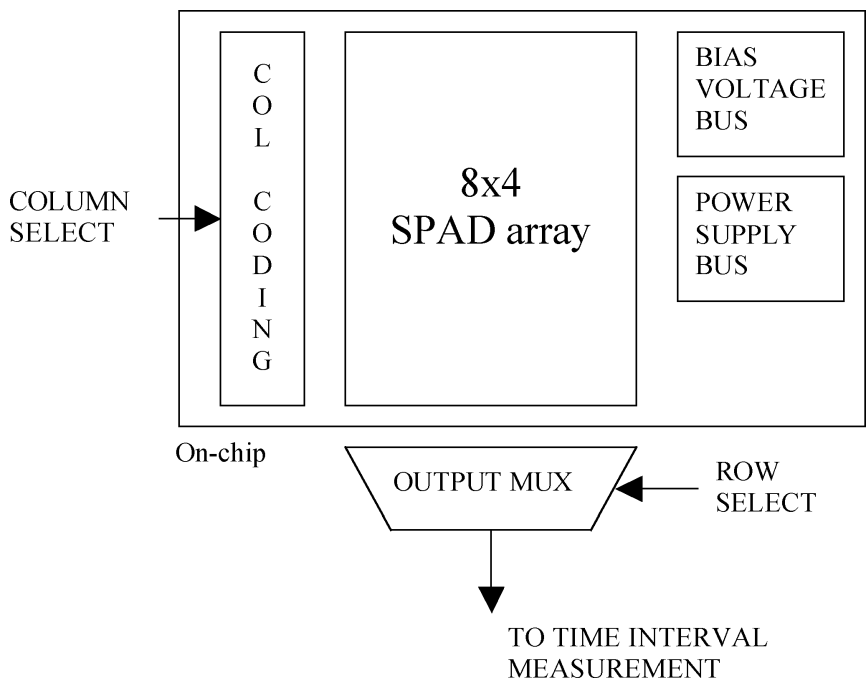

Fig. 4. Sensor architecture.

light beam was intentionally uncollimated to create a cone of diffused radiation that could cover the entire scene. The detector array was placed behind a standard camera objective. The time elapsed between the laser trigger (START) and the response of a SPAD (STOP) was measured using a FLUKE PM6681 counter with a time resolution of $50 \mathrm{ps}$ and a jitter less than 150 ps. A time histogram was captured for every SPAD independently, but in a sequential manner due to the discrete setup.

For a single measurement, the uncertainty $\sigma(d)$ is evaluated as $\sigma(d)=c \sigma(\tau) / 2$, where $\sigma(\tau)$ is the time measurement uncertainty. In order to achieve a resolution $R$ over distance $d$, one must be able to resolve TOF increments $\Delta \tau=2 R / c$. Note that $\Delta \tau$ must be equal or comparable to $\sigma(\tau)$ for the measurement to be meaningful. Our setup shows an uncertainty of $268 \mathrm{ps}$, mainly limited by the jitter of the external components. Therefore, in order to obtain subpicosecond precision, it is necessary to average over $M$ measurements, the precision scaling with the square root of $M$.

In the inset plot of Fig. 6 a histogram of $\tau$ (centered around zero) is shown after $M=10^{4}$ measurements. Call $h_{\tau}(t)$ such a histogram. Due to the various skews introduced by the layout of the array and external discrete components, it is advantageous to compute two histograms of $\tau$ for every pixel. The first $h_{\tau}^{\prime}(t)$ relates to the scene of interest, the second $h_{\tau}^{\prime \prime}(t)$ to a reference, for example a background panel. Note that the reference image needs to be taken only once and is subsequently stored electronically. Instead of computing an absolute time, we evaluate a TOF difference. This procedure is accomplished by numerically computing the centroid of the cross-correlation function between $h_{\tau}^{\prime}(t)$ and $h_{\tau}^{\prime \prime}(t)$. This method numerically simulates the presence of an optical copy of the source beam; however, a complex setup to generate such copy is avoided [6].

The rangefinding performance of the 3-D CMOS sensor was tested with $M=10^{4}$. From the histograms, $\sigma(\tau)$ was measured to be $268 \mathrm{ps}$, which yields a theoretical uncertainty $\sigma(d)=$ $402 \mu \mathrm{m}$ after $10^{4}$ acquisitions. Fig. 6 shows a plot of measured versus actual distance computed over a range of $15 \mathrm{~cm}$ around $1 \mathrm{~m}$. The standard deviation was $618 \mu \mathrm{m}$ throughout the entire 


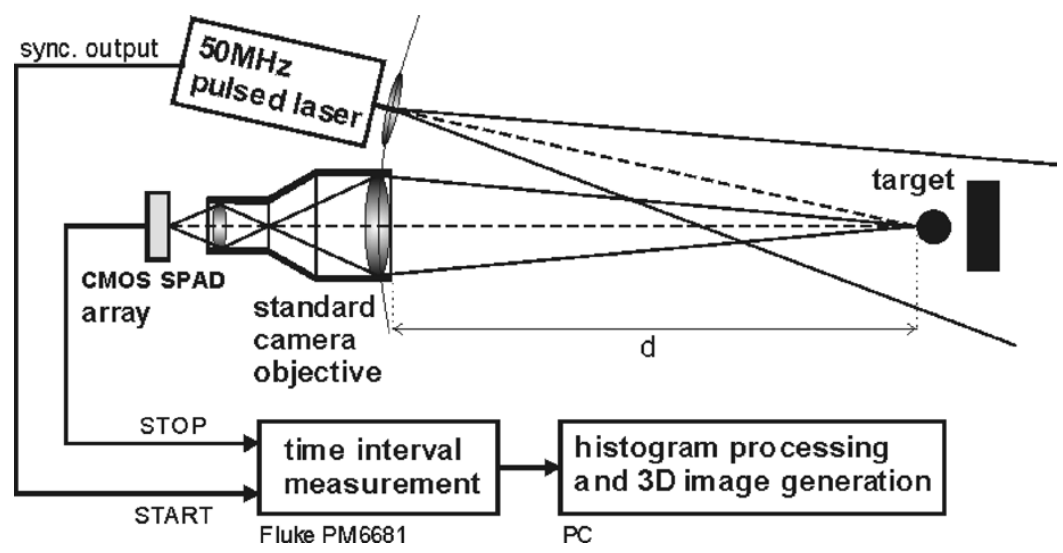

Fig. 5. 3-D camera setup.

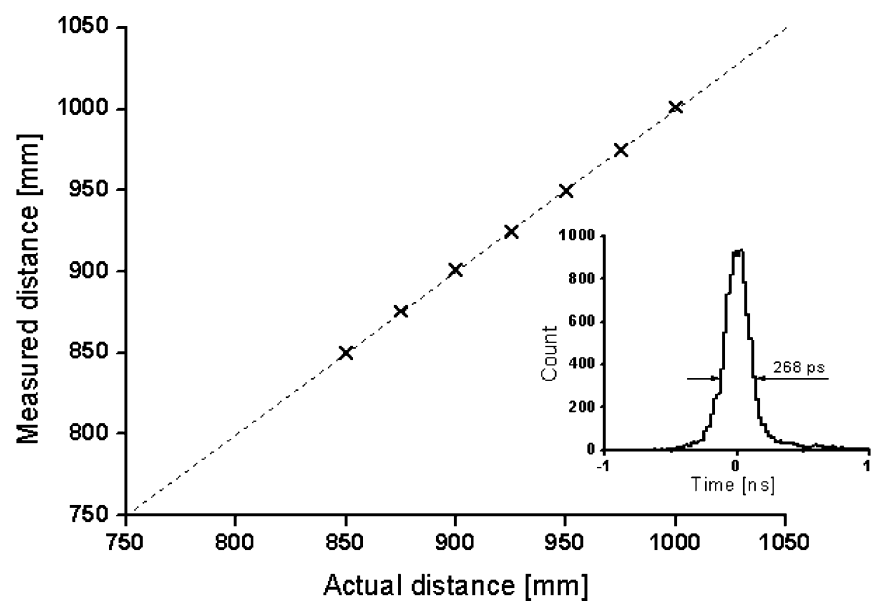

Fig. 6. Distance measurement. In inset: arrival time histogram centered around zero.

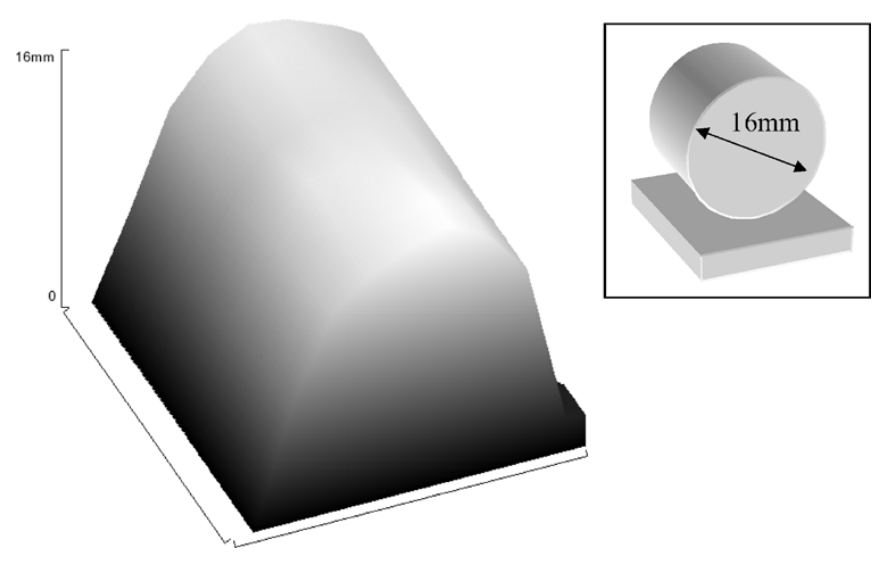

Fig. 7. Depth map of a cylinder.

range. The probable cause of the mismatch in error is the finite-time resolution of the counter, which results in histogram quantization noise.

The detector array was also used to extract the 3-D surface of several scenes. Figs. 7 and 8 show two such 3-D images extracted using the proposed algorithm. A submillimetric axial accuracy was obtained at a distance of $1 \mathrm{~m}$.

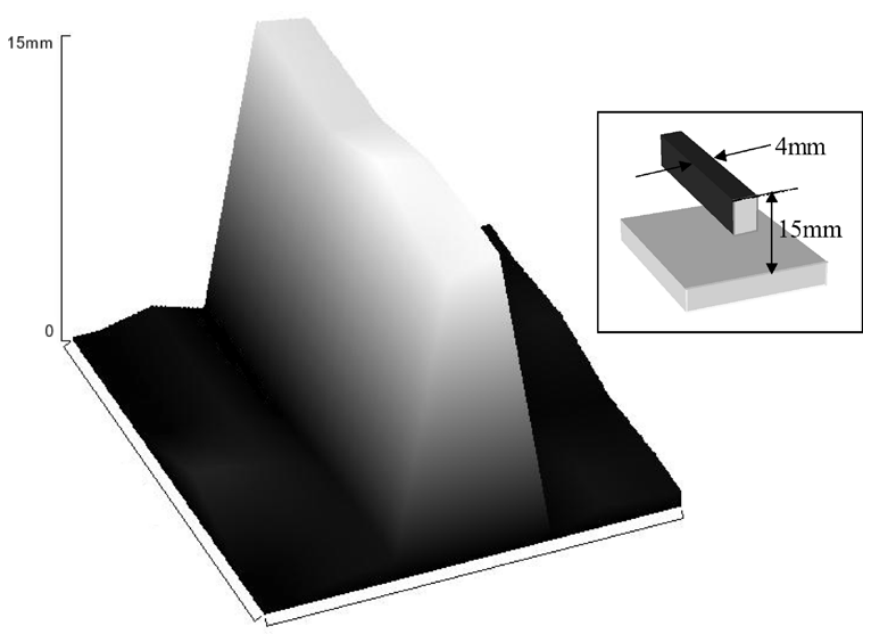

Fig. 8. Depth map of a suspended bar.

\section{Evaluation of SPAD Technology For 2-D GRAYSCALE IMAGERS}

Texture analysis could be used in 3-D applications to better evaluate the subjects in the scene. In the remainder of the paper, we establish the characteristics of a sensor based on SPADs used as 2-D grayscale imagers.

Critical features of standard 2-D grayscale imagers are temporal noise and saturation, which, in turn, determine the dynamic range. In this section, we study these features as a function of quantum efficiency, dark count rate (DCR), sensor dead time, and the optical intensity. The light source for 2-D imaging could be the same laser beam used in 3-D mode with or without modulation, or a separate continuous source. The electronics may not necessarily be identical to the one used in the 3-D mode.

\section{A. Temporal Noise}

Since Geiger pulses are digital, the classical analysis used in standard CMOS imagers does not apply. In SPADs the temporal noise is given by time-varying dark counts and photonic noise. Both sources of noise have a Poissonian nature. At low illumination intensity $I$, and for a given pixel acquisition time (PAT), we 


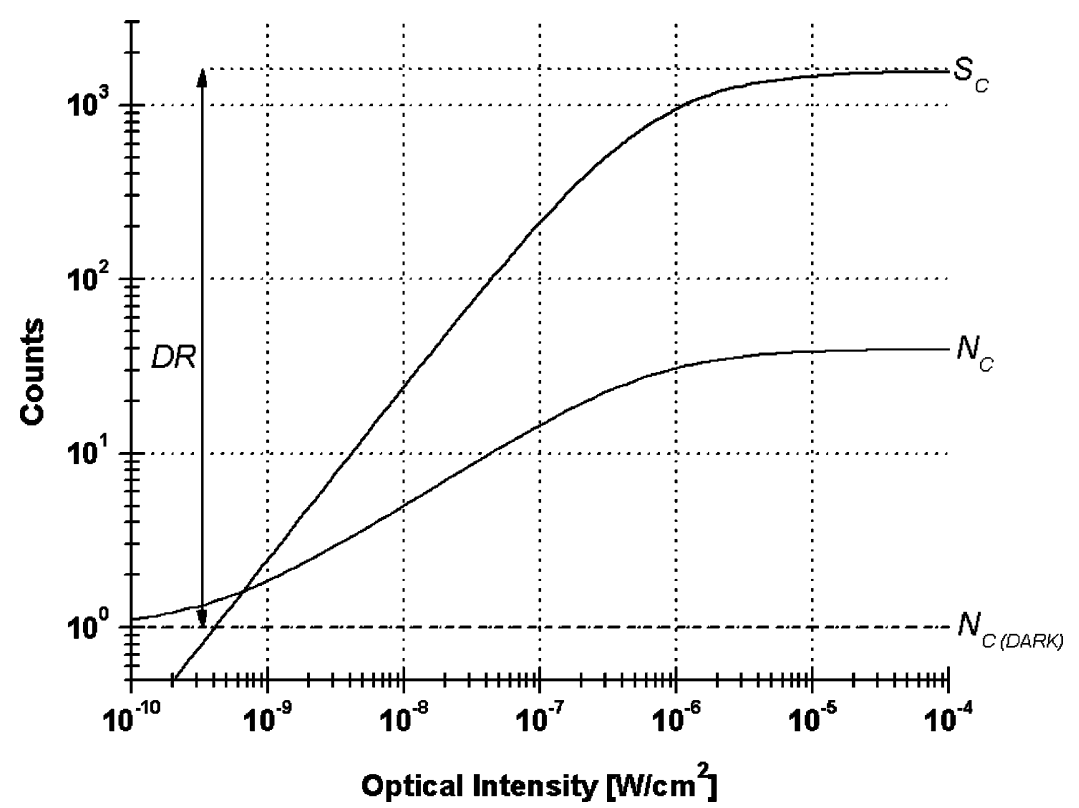

Fig. 9. Signal counts $S_{c}$ and noise counts $N_{c}$ as function of the optical intensity. $\left(\lambda=635 \mathrm{~nm}, \eta=10 \%, A=38 \mu \mathrm{m}^{2}, \mathrm{DCR}=50 \mathrm{~Hz}, N_{\mathrm{ROWS}}=128\right.$, $\mathrm{RBW}=10 \mathrm{MHz}$ and PAT $=20 \mathrm{~ms}$ ).

can relate the noise counts $N_{c}$ with the mean measured counts $n_{\text {eff }}$ by

$$
N_{c}^{2}=n_{\mathrm{eff}} \cong(\mathrm{DCR} \cdot \mathrm{PAT})+S_{c}
$$

where $S_{c}$ refers to the signal counts. The first term represents the fluctuation of the DCR resulting from the thermal and tunneling generation of carriers; the second term takes the signal shot noise into account. The signal counts under low illumination are expressed as

$$
S_{c}=\frac{\eta \lambda}{h c} \cdot I \cdot A \cdot \mathrm{PAT}
$$

where $\eta, \lambda$, and $A$ are quantum efficiency, wavelength, and detector active area, respectively. The minimum detectable signal (MDS) is given by a signal-to-noise ratio (SNR) equal to unity $\left(N_{c}=S_{c}\right)$. It is therefore given by

$$
S_{\mathrm{MDS}} \cong \frac{1+\sqrt{1+4 \mathrm{DCR} \cdot \mathrm{PAT}}}{2} .
$$

\section{B. Saturation Power and Dynamic Range}

Let us now consider the signal counts under strong illumination. Depending on the sensor architecture, the saturation behavior depends on several factors. We distinguish two important cases.

In the first case, the architecture could be capable of storing the light intensity on pixel (e.g., via a counter [5]). Such architecture would lead, however, to a strongly reduced fill factor.

In the second case, an integrated readout circuit could be shared between several pixels. Let us consider the case where the readout circuit is integrated for each column of the array.
Let $N_{\text {ROwS }}$ be the number of rows of the sensor, then the saturation signal count $\left(S_{\mathrm{SAT}}\right)$ of a pixel is

$$
S_{\mathrm{SAT}}=\frac{\mathrm{RBW}-\mathrm{DCR} \cdot \mathrm{N}_{\mathrm{ROWS}} \mathrm{PAT}}{\mathrm{N}_{\mathrm{ROWS}}}
$$

where RBW is the column readout bandwidth. Note that we consider the worst case in which the image is completely bright, while in a typical image some pixels may not be completely saturated, thus leaving their share of bandwidth available to other pixels on the same column.

In order to determine the equation for signal counts under strong illumination we have to take the dead time imposed by whole system into account. The ultimate limit of the column readout bandwidth is set by the detector dead time (RBW $\leq$ $1 / \delta)$. Therefore, the saturation behavior is only determined by the dead time of the readout circuitry $\left(\delta_{\mathrm{RO}}\right)$.

In this case, for an ideal sensor $\left(\delta=\delta_{\mathrm{RO}}=0\right)$, the mean observed counts $n_{\text {ideal }}$ are

$$
\frac{n_{\text {ideal }}}{\mathrm{PAT}}=\left(\frac{\eta \lambda}{h c} \cdot I \cdot A\right)+\mathrm{DCR} .
$$

For a real detector, the readout dead time reduces the time during which the counts can be recorded. To a first approximation, the effective acquisition time is

$$
\begin{aligned}
\Delta t_{\mathrm{eff}} & =\mathrm{PAT}-\left(n_{\mathrm{eff}} \cdot N_{\mathrm{ROWS}} \cdot \delta_{\mathrm{RO}}\right) \\
& =\mathrm{PAT}-\left(\frac{n_{\mathrm{eff}} \cdot N_{\mathrm{ROWS}}}{\mathrm{RBW}}\right) .
\end{aligned}
$$

Thus, the effectively measured counts are the product of the ideal count rate by the effective acquisition time

$$
n_{\text {eff }} \cong \frac{n_{\text {ideal }}}{\mathrm{PAT}} \cdot \Delta t_{\text {eff }} \cong n_{\text {ideal }} \cdot \frac{1}{1+\frac{n_{\text {ideal }}}{\text { PAT }} \frac{N_{\text {RoWs }}}{\text { RBW }}} .
$$


The signal counts become

$$
\frac{S_{c}}{\mathrm{PAT}}=\frac{n_{\mathrm{eff}}}{\mathrm{PAT}}-D C R \cong\left(\frac{\eta \lambda}{h c} I \cdot A\right) \cdot \frac{1}{1+\left(\frac{\eta \lambda}{h c} I \cdot A\right) \cdot \frac{N_{\mathrm{ROWS}}}{\mathrm{RBW}}}
$$

where the terms in DCR $\cdot \delta_{\mathrm{RO}}$ were neglected.

The dynamic range (DR) of an imager is defined as the maximum output swing divided by the temporal noise in the dark. Thus, using the above equations, it can be expressed as

$$
\begin{aligned}
\mathrm{DR} & =20 \log _{10}\left[\frac{S_{\mathrm{SAT}}}{\left.N_{C}\right|_{\mathrm{DARK}}}\right] \\
& \cong 20 \log _{10}\left[\frac{\mathrm{RBW}}{\mathrm{N}_{\text {ROWS }}}\left(\frac{\mathrm{PAT}}{\mathrm{DCR}}\right)^{\frac{1}{2}}\right] .
\end{aligned}
$$

\section{Numerical Results and Discussion}

Using the experimental values of Section II, signal and noise, as computed in (1) and (5), are plotted in Fig. 9. The PAT used for the estimation is $20 \mathrm{~ms}$, which corresponds to a frame rate of $50 \mathrm{fps}$. In addition, we consider $N_{\text {ROwS }}=128$ and $\mathrm{RBW}=10 \mathrm{MHz}$. These parameters can be easily achieved in this technology.

The minimum detectable signal $S_{\mathrm{MDS}}$ is two counts, corresponding to an optical intensity of $8.13 \times 10^{-10} \mathrm{~W} / \mathrm{cm}^{2}$. As shown by (4), the saturation signal $S_{\mathrm{SAT}}$ mostly depends on the readout bandwidth and the number of rows. A saturation signal of 1562 counts which corresponds to an optical intensity of $1.98 \times 10^{-3} \mathrm{~W} / \mathrm{cm}^{2}$ is estimated using the proposed parameters. Finally, a dynamic range of $64 \mathrm{~dB}$ is obtained. This makes SPAD arrays a promising alternative to conventional CMOS for highly sensitive 2-D grayscale imagers.

\section{CONCLUSION}

A camera capable of extracting the depth map from a 3-D scene is proposed. The imager is based on an array of single photon avalanche diodes operating in Geiger mode fabricated in standard CMOS technology. Extremely reduced time jitter enables submillimetric axial accuracy in 3-D scenes. The scenes were illuminated by a low-power $50-\mathrm{MHz} 635-\mathrm{nm}$ laser source with no requirement of mechanical scanning of any kind. Estimates of dynamic range, temporal noise, and saturation show a promising potential for this type of devices to be employed in 2-D mode as well.

\section{REFERENCES}

[1] S. Yoshimura, T. Sugiyama, K. Yonemoto, and K. Ueda, "A 48 kfram/s CMOS image sensor for real-time 3-D sensing and motion estimation," in Proc. ISSCC, Feb. 2001, pp. 94-95.

[2] B. Saleh and M. Teich, Fundamentals of Photonics. New York: Wiley, 1991.

[3] R. Lange, "3D time-of-flight distance measurement with custom solid-state image sensors in CMOS/CCD-technology," Ph.D. dissertation, ETH-Zürich, Switzerland, Sept. 2000.
[4] E. Charbon and C. Bamji, "Methods for CMOS-compatible three-dimensional image sensing using quantum efficiency modulation," U.S. Patent 6515740 , Feb. 2003.

[5] B. F. Aull et al., "Geiger-mode avalanche photodiodes for three dimensional imaging," Lincoln Lab. J., vol. 12, no. 2, pp. 335-350, 2002.

[6] J. Massa, G. Buller, A. Walker, G. Smith, S. Cova, M. Umasuthan, and A. M. Wallace, "Optical design and evaluation of a three-dimensional imaging and ranging system based on time-correlated single-photon," Appl. Optics, vol. 41, no. 6, pp. 1063-1070, Feb. 2002.

[7] R. Jeremias, W. Brockherde, G. Doemens, B. Hosticka, L. Listl, and P. Mengel, "A CMOS photosensor array for 3D imaging using pulsed laser," in Proc. ISSCC, Feb. 2001, pp. 252-253.

[8] M. L. Perkins, S. J. Hill, S. P. Mickan, D. Abbott, J. Munch, and T. vanDoorn, "Review of tradeoffs for quenched avalanche photodiode sensors for imaging turbid media," Microelectron. J., vol. 31, no. 7, pp. 605-610, 2000.

[9] A. Rochas, "Single photon avalanche diodes in CMOS technology," Ph.D. dissertation, EPFL, 2003.

[10] A. Rochas, A. R. Pauchard, P. A. Besse, D. Pantic, Z. Prijic, and R. S. Popovic, "Low-noise silicon avalanche photodiodes fabricated in conventional CMOS Technologies," IEEE Trans. Electron Devices, vol. 49, pp. 387-394, March 2002

[11] A. Rochas, M. Gösch, A. Serov, P. A. Besse, R. S. Popovic, T. Lasser, and R. Rigler, "First fully integrated 2-D array of single-photon detectors in standard CMOS technology," IEEE Photon. Technol. Lett., vol. 15, pp. 963-965, July 2003.

[12] C. Niclass, A. Rochas, P. A. Besse, and E. Charbon, "A CMOS single photon avalanche diode array for 3D imaging," in Proc. ISSCC, Feb. 2004, pp. 120-121.

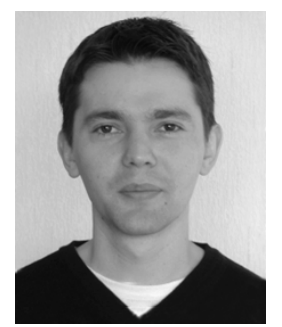

Cristiano Niclass received the M.S. degree in microengineering from the Swiss Federal Institute of Technology, Lausanne (EPFL), Switzerland, in 2003. He is currently working toward the Ph.D. degree in electrical and computer engineering at EPFL.

$\mathrm{He}$ is a Research Assistant in the Processor Architecture Laboratory (LAP). His research interests include the design of digital and mixed-mode integrated circuits. He is currently in charge of the design and implementation of a fully integrated two- and three-dimensional CMOS imager sensor based on single photon avalanche diodes.

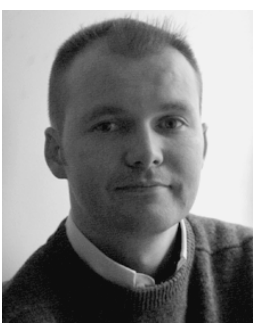

Alexis Rochas was born in Lyon, France, in 1971. In 1995, he received the M.S. degree in physics at the University Lyon 1, France. In 1997, he received the Postgraduate Diploma in control sciences and technologies from the Institute of Vision Engineering, Saint-Etienne, France. He received the Ph.D. degree from the Swiss Federal Institute of Technology Lausanne (EPFL), Switzerland, in 2003.

As a Research Engineer, he worked for Crismatec, a Saint-Gobain subsidiary, France, and was in charge of reducing the production costs of optical crystals. In 1998, he joined the Institute of Microsystems of the EPFL, as a Research Assistant. He worked on the development of an UV-sensor microsystem for flame detection in the framework of an industrial collaboration with Siemens Building Technologies, Inc. During his thesis work, he developed the first solid-state array of SPADs with integrated electronics. From June 2000, he worked as a Ph.D. student in the field of single photon avalanche diodes (SPADs) fabricated in standard CMOS technology. He also developed dedicated single-photon detectors in collaboration with Siemens Building Technologies for flame detection application, with Gnothis SA, Lausanne, Switzerland, for single-molecule detection by fluorescence correlation spectroscopy, and with IdQuantique, Geneva, Switzerland, for random numbers generation. In November 2003, he joined the Processor Architecture Laboratory of EPFL where he is in charge of designing a large array of SPADs in CMOS technology and investigating potential applications. 


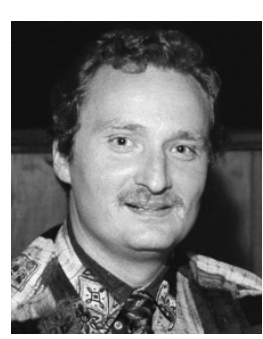

Pierre-André Besse was born in Sion, Switzerland, in 1961 . He received the diploma in physics and $\mathrm{Ph} . \mathrm{D}$. degree in semiconductor optical amplifiers from the Swiss Federal Institute of Technology, ETH, Zurich, Switzerland, in 1986 and 1992, respectively.

In 1986, he joined the group of micro- and optoelectronics of the Institute of Quantum Electronics at ETH Zurich, where he is engaged in research in optical telecommunication science. He worked on theory, modeling, characterization, and fabrication of compound semiconductor devices. In August 1994, he joined the Institute of Microsystems at the Swiss Federal Institute of Technology, Lausanne, Switzerland, as a Senior Assistant, where he starts activities on sensors and actuators microsystems. His research interests include physical principles and new phenomena for optical, magnetic, and inductive sensors. He has written and coauthored over 100 scientific papers and conference contributions.

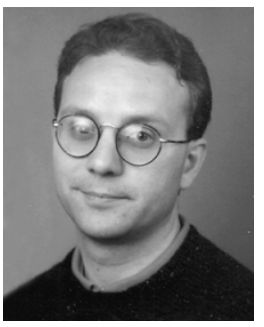

Edoardo Charbon (M'92) received the Diploma in electrical engineering from the Swiss Federal Institute of Technology (ETH), Zurich, Switzerland, in 1988, the M.S. degree in electrical and computer engineering from the University of California, San Diego, in 1991, and the Ph.D. degree from the University of California, Berkeley, in 1995. His doctoral work focused on performance-directed constraint-based analog and mixed-signal physical design automation and accelerated substrate extraction techniques.

From 1995 to 2000, he was with Cadence Design Systems, where he was the architect of the company's initiative for intellectual property protection. In 2000, he joined Canesta, Inc., as its Chief Architect, leading the development of wireless three-dimensional CMOS image sensors. Since November 2002, he has been a member of the Faculty of the Swiss Federal Institute of Technology, Lausanne, Switzerland, working in the field of three-dimensional (3-D) sensors and ultralow-power wireless embedded systems. He has consulted for numerous organizations, including Texas Instruments Incorporated, Hewlett-Packard, and the Carlyle Group. He has published over 55 articles in technical journals and conference proceedings and two books, and he holds six patents. His research interests include 3-D micro-imaging, radio-frequency integrated circuits, intellectual property protection, and substrate modeling and characterization.

Dr. Charbon has served as a Guest Editor of the IEEE TRANSACTIONS ON COMPUTER-AIDED DESIGN OF INTEGRATED CIRCUITS AND SYSTEMS and IEEE Journal of Solid StATE Circuits. He has also been a Member of the Technical Committee of the IEEE Custom Integrated Circuits Conference since 1999. 